\section{Plant Metabolomics - Strategies for Biomarker Detection, Isolation, and Identification}

Elia Grata ${ }^{\mathrm{ab}}$, Julien Boccard ${ }^{\mathrm{bc}}$, Gaëtan Glauser ${ }^{\mathrm{ab}}$, Davy Guillarme $^{\mathrm{a}}$, Pierre-Alain Carrupt ${ }^{\mathrm{c}}$, Jean-Luc Wolfender ${ }^{\mathrm{b}}$, and Serge Rudaz ${ }^{* a}$

${ }^{*}$ Correspondence: Dr. S. Rudaza, Tel.: +41 2237965 72, Fax: +4122 37968 08, E-Mail: serge. rudaz@pharm.unige.ch

aLCAP, bLPP, CLCT, Ecole de Pharmacie Genève-Lausanne, Section des Sciences Pharmaceutiques, Université de Genève, Université de Lausanne, $\mathrm{CH}-1211$ Genève

Keywords: Arabidopsis thaliana - Capillary NMR - Data mining - Metabolomics · Plant metabolism - Stress-induced metabolites · UPLCTOF-MS

Recent developments in analytical methods and data mining have permitted metabolomics to evolve from an ambitious concept to a valuable technology which provides a global picture of molecular organisation at the metabolite level.

A strategy was developed for the detection, isolation, and identification of stress-induced metabolites produced in Arabidopsis thaliana after wounding the leaves, which mimics the herbivore attack. Although several defence signalling compounds are identified, the expression of some of the defence genes is probably dependent on original compounds, which still need to be characterized. Therefore, the structure determination of these biomarkers represents an important analytical challenge since they are only found in minute amounts in plants, occur as closely related isomers and are convoluted with major constitutive plant secondary metabolites.

The developed metabolomic approach was based on a sequential strategy:

1) High-throughput metabolite fingerprinting involving rapid UPLC-TOF-

MS gradients on numerous wounded and unwounded leaf samples.

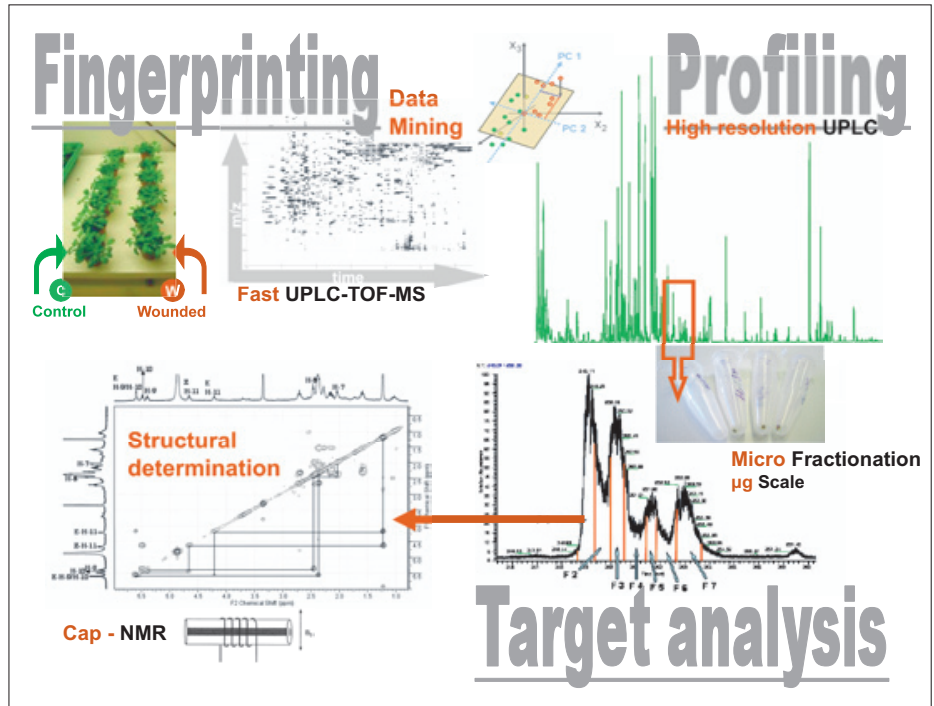

Analytical steps for the identification of stress biomarkers in plants
2) Data mining for group discrimination and determination of peaks responsible for the main metabolome variations.

3) High-resolution metabolite profiling of selected pool samples on high peak capacity UPLC columns after efficient gradient transfer for the localisation and deconvolution of the selected ions.

4) Targeted LC-MS triggered microfractionation of the biomarkers at the semi-preparative level based on computed LC conditions from UPLC gradients.

5) Complete structural determination of the unknown compounds based on at-line capillary-NMR experiments at the microgram level.

Thank to this strategy a broad survey of wound-biomarkers with various physicochemical properties was obtained and, besides known signalling molecules, original oxylipins and related products (jasmonates) were identified. This approach provides a rapid estimation of the significant wound metabolome variations, the identification of biomarkers involved in these changes and detailed information on their temporal and spatial dynamics.

\section{Acknowledgements}

The Swiss National Science Foundation (grant $\mathrm{n}^{\circ}$ 205320-116274/1 to J.L Wolfender and S. Rudaz) is thanked for supporting this work.

\section{References}

Received: May 10, 2008

J. Boccard, E. Grata, A. Thiocone, J. Y. Gauvrit, P. Lanteri, P.-A. Carrupt, J. L. Wolfender, S. Rudaz, Chemometr. Intell. Lab. Syst. 2007, 86, 189.

G. Glauser, D. Guillarme, E. Grata, J. Boccard, A. Thiocone, P.-A. Carrupt, J. L. Veuthey, S. Rudaz, J. L. Wolfender, J. Chromatogr. A 2007, 1180, 90.

E. Grata, J. Boccard, G. Glauser, P.-A. Carrupt, E. E. Farmer, J. L. Wolfender, S. Rudaz, J. Sep. Sci. 2007, 30, 2268.

G. Glauser, E. Grata, L. Dubugnon, S. Rudaz, E. Farmer, J. L. Wolfender, J. Biol. Chem. 2008, 283, 16400.

E. Grata, J. Boccard, D. Guillarme, G. Glauser, P.-A. Carrupt, E. E. Farmer, J. L. Wolfender, S. Rudaz, J. Chromatogr. B 2008, 871, 261.
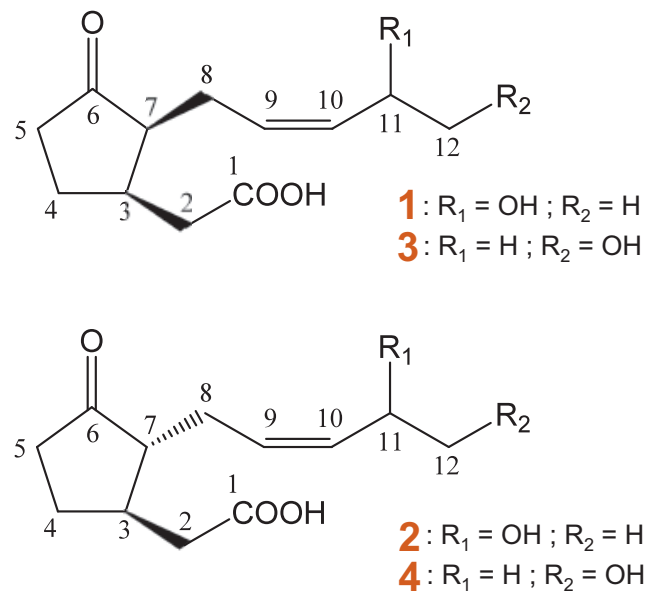

Jasmonic acid derivatives identified as wound biomarkers 